\title{
UNIVERSA MEDICINA
}

\section{Hepatic enzyme concentrations as indicators of nonalcoholic fatty liver disease}

\begin{abstract}
Alvina*
ABSTRACT

Nonalcoholic fatty liver disease (NAFLD) has emerged as a world-wide problem because it runs an asymptomatic course, ultimately leading to cirrhosis of the liver and portal hypertension, resulting in death. The prevalence of the disease accounts for $3-24 \%$ of the population in several countries. Generally there are increased concentrations of hepatic enzymes as markers of liver damage, such as serum glutamic oxaloacetic transaminase (SGOT), serum glutamic pyruvic transaminase (SGPT) and gamma glutamyl transferase (GGT). The aim of the present study was to determine the concentrations of hepatic enzymes as markers of NAFLD. The study design was cross-sectional, involving 90 subjects meeting the inclusion and exclusion criteria. The degree of severity NAFLD was determined by ultrasonography and the concentrations of SGOT, SGPT and GGT by automated clinical chemistry analyzer. The results indicated that there were 32 subjects with mild NAFLD (35.6\%), 35 subjects with moderate NAFLD (38.9\%) and 23 subjects with severe NAFLD (25.6\%). There was a significant difference in degree of NAFLD by gender $(\mathrm{p}<0.05)$, where severe NAFLD was more frequent in males than in females. Concentrations of SGOT, SGPT and GGT were significantly different between degrees of NAFLD $(\mathrm{p}<0.05)$. The conclusion is that SGOT, SGPT and GGT concentrations are indicators of degree of NAFLD.
\end{abstract}

Keywords: Nonalcoholic fatty liver disease, liver enzyme concentrations, ultrasonography
*Department of Clinical Pathology, Medical Faculty, Trisakti University

\section{Correspondence}

dr. Alvina, SpPK

Department of Clinical

Pathology, Medical Faculty,

Trisakti University

Jl. Kyai Tapa No.260

Grogol - Jakarta 11440

Phone: 021-5672731 ext.2404

Email:

vina_march_dr@yahoo.com

Univ Med 2009;28:139-45

\section{INTRODUCTION}

Nonalcoholic fatty liver disease (NAFLD) is a pathological clinical condition with a wide spectrum, including steatosis and steatohepatitis, with a potential for cirrhosis and hepatoma. The incidence of NAFLD varies with ethnicity or culture and with gender. ${ }^{(1)}$ According to the American Association for the Study of Liver Disease, NAFLD is a condition characterized by excessive accumulation of fat in the liver of 5$10 \%$ of the weight of the liver and the presence 
of fat-containing hepatocytes on microscopic examination. ${ }^{(2)}$

NAFLD is a sufficiently important problem because it runs an insidious asymptomatic course and ultimately causes cirrhosis of the liver and portal hypertension, culminating in death. ${ }^{(3)}$ The prevalence of NAFLD has been reported to account for $3-24 \%$ of the population of several countries, in parallel with the concomitant increase in the prevalence of diabetes and obesity, while the disease is the leading cause of liver disease in Western countries. ${ }^{(4-6)}$ NAFLD is also associated with type 2 diabetes, as $70 \%$ of patients with type 2 diabetes has steatosis, as determined by ultrasonography. ${ }^{(7)}$ In addition, visceral obesity in adults is considered to be the main risk factor for developing steatosis. ${ }^{(1)}$

The majority of patients with NAFLD are asymptomatic, although commonly having elevated circulatory concentrations of markers of hepatic damage, such as serum glutamic oxaloacetic transaminase (SGOT), serum glutamic pyruvic transaminase (SGPT) and gamma glutamyl transferase (GGT) ${ }^{(4,8)}$ SGPT is closely associated with accumulation of fat in the liver, such that in epidemiological studies SGPT is commonly used as surrogate marker of NAFLD. Elevated concentrations of SGPT are frequently associated with obesity and the metabolic syndrome. ${ }^{(4,9,10)}$ GGT is considered to be a sensitive but nonspecific indicator of hepatic damage and is the main component of intracellular antioxidant defenses. The association of GGT with diabetes is due to hepatic dysfunction as a result of fatty infiltration of the liver and this effect is considered to be capable of increasing oxidative stress. ${ }^{(11)}$

On the basis of the above mentioned discussion, the purpose of the present study was to determine the concentrations of hepatic enzymes as markers of NAFLD.

\section{METHODS}

\section{Research design}

The study was designed as a cross-sectional study, conducted from April to September 2008 in Hospital X in Jakarta.

\section{Study subjects}

The study subjects were patients with NAFLD, chosen consecutively from outpatients visiting the Hepatology clinic of Hospital X and meeting the following inclusion criteria: male and females aged 20-66 years, suffering from NAFLD as proven by ultrasonography (USG). The exclusion criteria were alcoholics and/or patients with hepatitis B and/or hepatitis C. On USG examination, NAFLD may be graded into three stages, namely mild, moderate, and severe. ${ }^{(12)}$ In mild NAFLD there is hepatomegaly with increased echogenicity of the hepatic parenchyma, designated as bright liver, where the diaphragmatic dome and all hepatic vascular structures are clearly visualized. In moderate NAFLD, in addition to increasing echogenicity of the hepatic surface, there is decreased echogenicity of the parenchyma of the subsurface areas, the diaphragm is somewhat hypoechogenic and the peripheral hepatic vascular structures become increasingly difficult to recognize. In severe NAFLD, the echogenicity of the hepatic surface is enhanced and most of the subsurface hepatic parenchyma is hypoechogenic whilst the diaphragm and the hepatic vascular structures are difficult to identify.

\section{Measurements}

The specimens to be examined, consisting of $5 \mathrm{~mL}$ venous blood taken from the cubital vein were collected in tubes without anticoagulant. The serum was separated from the blood maximally one hour after collection. The blood 
was centrifuged at $3000 \mathrm{rpm}$ for 10 minutes, then the serum was put in a sample cup for determination of the SGOT, SGPT and GGT activities. SGOT and SGPT levels were determined by the IFCC method without pyridoxal phosphate activation, whereas determination of GGT was by the Szasz method using 66-3' carboxy-4-nitranilide at $37^{\circ} \mathrm{C}$. The determinations were performed with a Hitachi 912 analyzer.

\section{Data analysis}

The collected data were analyzed by means of SPSS version 11.5. Chi-square tests were done to analyze the difference of degree of NAFLD between genders. To compare the hepatic enzyme concentration between degrees of NAFLD, analysis of variance (ANOVA) was performed. All the reported $\mathrm{p}$ values were 2tailed, and values of $\mathrm{p}<0.05$ were considered to be statistically significant.

\section{RESULTS}

The study involved 90 persons, consisting of 28 males (31.1\%) and 62 females (68.9\%), within the age range of 20-66 years. On USG examination, there were 32 subjects with mild NAFLD (35.6\%), 35 subjects with moderate NAFLD (38.9\%) and 23 subjects with severe NAFLD (25.6\%).

Findings for risk factors for NAFLD were as follows: 53 subjects with one risk factor (58.9\%), 31 subjects with two risk factors $(34.4 \%)$ and 6 subjects with three risk factors $(6.7 \%)$. In subjects with one risk factor, the most frequently encountered risk factor was hypercholesterolemia $(15.5 \%)$, followed in descending order of frequency by diabetes mellitus (14.4\%) and obesity (10.0\%). In subjects with two risk factors, there were combinations of risk factors, such as obesity and hypercholesterolemia, obesity and diabetes mellitus, diabetes mellitus and
Table 1. Distribution of degrees and risk factors of NAFLD

\begin{tabular}{lc}
\hline Variable & $\mathbf{n}(\boldsymbol{\%})$ \\
\hline Degree of NAFLD & $32(35.6)$ \\
Mild & $35(38.9)$ \\
Moderate & $23(25.6)$ \\
Severe & \\
NAFLD risk factors & $11(12.2)$ \\
Dyslipidemia & $13(14.4)$ \\
DM & $14(15.5)$ \\
Hypercholesterolemia & $4(4.4)$ \\
Hypertriglyceridemia & $2(2.2)$ \\
Hypertension & $9(10)$ \\
Obesity & $3(3.3)$ \\
Dyslipidemia + Hypertension & $5(5.5)$ \\
Dyslipidemia + DM & $3(3.3)$ \\
DM + Hypercholesterolemia & $1(1.1)$ \\
DM + Hypertriglyceridemia & $10(11.6)$ \\
DM + Hypertension & $1(1.1)$ \\
Hypercholesterolemia + Hypertension & $2(2.2)$ \\
Obesity + Dyslipidemia & $1(1.1)$ \\
Obesity + DM & $5(5.5)$ \\
Obesity + Hypercholesterolemia & $6(6.6)$ \\
Three risk factors* & \\
\hline H & \\
\hline -hypertion-dysipidema &
\end{tabular}

*DM-hypertension-dyslipidemia and obesity-DM-hypertension

hypertension. In subjects with three risk factors, the combinations were diabetes mellitushypertension-dyslipidemia and diabetes mellitushypertension-obesity (Table 1).

Among the subjects with mild NAFLD there were 9 males $(28.1 \%)$ and 23 females $(71.9 \%)$. Subjects with moderate NAFLD comprised 7 males $(20 \%)$ and 28 females $(80 \%)$ and those with severe NAFLD consisted of 12 males $(52.2 \%)$ and 11 females (47.8\%). The Chi-square test by gender and degree of NAFLD demonstrated a significant difference in degree of NAFLD between males and females $(\mathrm{p}<0.05)$. The proportion of severe NAFLD in males $(42.9 \%)$ was significantly higher than in females (17.7\%), as may be seen from Table 2. A subtantial proportion of the subjects $(36.0 \%)$ was in the age group of $\geq 50$ years. However, the 
Table 2. Characteristics of the study participants by degree of NAFLD

\begin{tabular}{|c|c|c|c|c|}
\hline \multirow[b]{2}{*}{ Characteristics } & \multicolumn{3}{|c|}{ Degree of NAFLD } & \multirow[b]{2}{*}{$\mathbf{p}$} \\
\hline & $\begin{array}{c}\text { Mild } \\
(n=32)\end{array}$ & $\begin{array}{c}\text { Moderate } \\
(\mathbf{n}=35)\end{array}$ & $\begin{array}{c}\text { Severe } \\
(23) \\
\end{array}$ & \\
\hline \multicolumn{5}{|l|}{ Age (years) } \\
\hline $20-29$ & $1(12.5 \%)$ & $2(25.0 \%)$ & $5(62.5 \%)$ & 0.715 \\
\hline $30-39$ & $5(29.4 \%)$ & $9(52.9 \%)$ & $3(17.6 \%)$ & \\
\hline $40-49$ & $12(40.0 \%)$ & $12(40.0 \%)$ & $6(20.0 \%)$ & \\
\hline$\geq 50$ & $14(40.0 \%)$ & $12(34.3 \%)$ & $9(25.7 \%)$ & \\
\hline \multicolumn{5}{|l|}{ Geñder } \\
\hline Male & $9(32.1 \%)$ & $7(25.0 \%)$ & $12(42.9 \%)$ & $0.037 *$ \\
\hline Female & $23(37.1 \%)$ & $28(45.2 \%)$ & $11(17.7 \%)$ & \\
\hline
\end{tabular}

* Significant $(\mathrm{p}<0.05)$

majority of subjects $(62.5 \%)$ with severe NAFLD was in the age group of 20-29 years.

In mild NAFLD the mean concentrations of SGOT, SGPT and GGT were 19.1 U/L, $18.1 \mathrm{U} /$ $\mathrm{L}$, and $23.5 \mathrm{U} / \mathrm{L}$, respectively. In moderate NAFLD mean SGOT concentration was $26.2 \mathrm{U} /$ L, while mean SGPT was 27.1 U/L and mean GGT 45.2 U/L. In severe NAFLD mean SGOT, SGPT and GGT activities were respectively 58.0 U/L, 64.1 U/L, and 60.7 U/L. ANOVA results showed SGOT, SGPT and GGT activities to be significantly elevated in proportion to degree of NAFLD $(\mathrm{p}<0.05)$ as indicated in Table 3.

\section{DISCUSSION}

In the present study, among the 90 study subjects there were a higher number of persons with NAFLD of mild and moderate degree (35.6\% and $38.9 \%$, respectively), compared with those with NAFLD of severe degree. These findings may have been due to the fact that in the present study no distinction was made between subjects with newly acquired NAFLD and those who had recovered from NAFLD of severe degree. Thus the possibility exists that subjects with NAFLD of severe degree made a recovery after treatment and thus were entered into the study as NAFLD of moderate or mild degree.

In this study the number of female subjects was higher than that of males, which is supported by the study of De Lusong et al. ${ }^{(14)}$ in the Philippines in 2005 where it was also found that female NAFLD patients amounted to $71 \%$ of the 134 study subjects. ${ }^{(13)}$ In contrast, Amarapurkar et al. in India found more males with NAFLD than females $(24.6 \%$ vs $13.6 \%)$. The reason why NAFLD is found more frequently in males than in females may be due to an excess of intraabdominal fat in males, and possibly also to the fact that the distribution of body fat is influenced

Table 3. SGOT, SGPT and GGT activities in NAFLD

\begin{tabular}{ccccc}
\hline Enzyme & Mild NAFLD & Moderate NAFLD & Severe NAFLD & p \\
\hline SGOT (U/L) & $19.1 \pm 7.3$ & $26.2 \pm 10.2$ & $58.0 \pm 41.5$ & $0.00^{*}$ \\
SGPT (U/L) & $18.1 \pm 6.9$ & $27.1 \pm 13.1$ & $64.1 \pm 48.6$ & $0.00^{*}$ \\
GGT (U/L) & $23.5 \pm 10.1$ & $45.2 \pm 27.2$ & $60.7 \pm 35.3$ & $0.00^{*}$ \\
\hline
\end{tabular}

* Significant $(\mathrm{p}<0.05)$ 
by sex hormones. Sex hormone binding globulin (SHBG) synthesized in the liver is strongly correlated with insulin sensitivity. ${ }^{(15)}$

The age range of the subjects in the present study was 20-66 years. In another study in Pakistan the age was between 47 and 54 years. ${ }^{(16)}$ Another investigator also found that NAFLD was more common in the younger age groups, which may have been due to changes in dietary pattern and the habit of consuming soft drinks, as revealed by another study indicating that consumption of soft drinks was associated with an elevated risk of NAFLD, although the precise mechanism is unclear. ${ }^{(17)}$ According to Shibata, the rapid rise of NAFLD in the younger age groups is the result of a westernized life style, such as is reflected by a high-fat and high-calorie diet and a decline in physical activity. ${ }^{(18)}$ These results provide confirmatory evidence for our study, where severe NAFLD was most frequent in the age group of 20-29 years.

The most frequent risk factors of NAFLD in the present study were hypercholesterolemia, diabetes mellitus, dyslipidemia, and obesity. The study found the cause of NAFLD to be the metabolic syndrome, i.e. hyperlipidemia $(80.6 \%)$, obesity $(37 \%)$ and type 2 diabetes mellitus (18\%). ${ }^{(19)}$ The metabolic syndrome is associated with insulin resistance (IR), which in turn is related to elevated synthesis of free fatty acids from glucose in the liver. In addition, adipose tissue is a source of tumor necrosis factor-á (TNF-á), which is a regulator of insulin sensitivity. TNF-á may increase insulin resistance by depressing peroxisome proliferator activated receptor gamma (PPARã), which is an important receptor for maintaining insulin sensitivity within normal limits. ${ }^{(20,21)}$ Generally the number of NAFLD patients increase in proportion to the course of the metabolic syndrome and insulin resistance. The metabolic syndrome is a collection of signs and symptoms consisting of diabetes, central obesity, hypertension and dyslipidemia. ${ }^{(22,23)}$ In obese patients there is an increased uptake of free fatty acids by the liver, whereafter the acids are subjected to â-oxidation or esterification with glycerol to form triglycerides that play a role in the accumulation of fat in the liver. ${ }^{(24)}$

The study by Chang et al. ${ }^{(25)}$ showed an association between SGPT and the incidence of NAFLD as determined by USG. Therefore it may be concluded that elevated SGPT is an independent predictor of NAFLD events. Because SGPT is a glucogenic enzyme, elevated SGPT is also an indicator of insulin abnormalities. ${ }^{(26)}$ NAFLD patients have higher SGPT concentrations in comparison with SGOT, and is mainly synthesized in the liver, making SGPT more specific for liver disease. ${ }^{(27,28)}$ An elevated SGPT concentration in asymptomatic individuals is considered of value as a screening test for NAFLD patients, as SGPT concentrations are correlated with abdominal fat and as higher SGPT concentrations are associated with the degree of steatosis..$^{(1,29,30)}$

The present study also found elevated GGT concentrations proportional to the degree of NAFLD, thus it may be assumed that GGT is correlated with NAFLD. The precise mechanisms of the correlation of GGT with NAFLD is still unclear. However, the explanation suggested by Grundy et al. ${ }^{(31)}$ is that accumulation of fat in the liver probably increases oxidative stress, leading to increased consumption of glutathione (GSH). This is compensated for by increased GGT synthesis, as GGT plays a role in glutathione catabolism. GGT is also involved in the pathogenesis of diabetes through another mechanism associated with oxidative stress. The association between GGT and diabetes is indicated by continued exposure to organic pollutants, where these latter may interact with obesity, thus resulting in diabetes. ${ }^{(11)}$ 
Elevated hepatic enzyme concentrations may indicate the presence of excessive fat deposits in the liver, which are indirectly associated with obesity and deposits of visceral fat, now considered as part of the metabolic syndrome. ${ }^{(11)}$ According to Tiikkainen et al., elevated liver enzymes are correlated with increases in hepatic fat and NAFLD. ${ }^{(32)}$ According to Thamer et al. SGOT and GGT concentrations are also correlated with hepatic fat independently of obesity, although SGOT is less sensitive than SGPT. ${ }^{(33)}$

The present study has several limitations. First, the steatosis was determined by ultrasonography instead of liver biopsy as the gold standard. However, ultrasonography is sensitive, non-invasive and practical for determining NAFLD. But ultrasonography cannot accurately differentiate steatosis from fibrosis. ${ }^{(34)}$ Second, the study participants were mostly persons on medical checkup and thus less representative of the general population. Third, the sample size was too small. NAFLD is generally asymptomatic and it is only the development of cirrhosis that has clinical consequence. At present, therapy in NAFLD concentrates on managing risk factors but in the future clinical trials may provide robust evidence for the use of insulin sensitising agents and other potential therapies. ${ }^{(35)}$

\section{CONCLUSIONS}

In patients with NAFLD the increase in SGOT, SGPT and GGT concentrations were independent indicators of the degree of NAFLD.

\section{REFERENCES}

1. Rocha R, Cotrim HP, Bitencourt AGV, Barbosa DBV, Santos AS, Almeida ADM, et al. Nonalcoholic fatty liver disease in asymptomatic
Brazilian adolescents. World J Gastroenterol 2009;15:473-7.

2. Kotronen A, Yki-Jarvinem H. Fatty liver: a novel component of the metabolic syndrome. Arterioscler Thromb Vasc Biol 2008;28:27-38.

3. Ahmed MH. The road map for the diagnosis of nonalcoholic fatty liver disease. Am J Clin Pathol 2007; 127:20-2.

4. Chen ZW, Chen LY, Dai HI, Chen JH, Fang LZ. Relationship between alanine aminotransferase levels and metabolic syndrome in nonalcoholic fatty liver disease. J Zhejiang Univ Sci B 2008;9:61622.

5. Clark JM. The epidemiology of nonalcoholic fatty liver disease in adults. J Clin Gastroenterol 2006;40 Suppl:S5-10.

6. Ong J, Younossi Z. Epidemiology and natural history of NAFLD and NASH. Clin Liver Dis 2007;11:1-16.

7. Targher G, Bertolini L, Padovani R, Rodella S, Tessari R, Zenari L. Prevalence of nonalcoholic fatty liver disease and its association with cardiovascular disease amongtype 2 diabetic patients. Diabetes Care 2007;30:1212-8.

8. Clark J, Diehl A. Defining nonalcoholic fatty liver disease: implications for epidemiologic studies. Gastroenterology 2003;124:248-50.

9. Marchesini G, Avagnina S, Barantani E, Ciccarone A, Corica F, Dalle G, et al. Aminotransferase and gamma glutamyltranspeptidase levels in obesity are associated with insulin resistance and the metabolic syndrome. J Endocrinol Invest 2005;28:333-9.

10. Schindhelm R, Diamant M, Dekker J, Tushuizen M, Teerlink T, Heine R. Alanine aminotransferase as a marker of nonalcoholic fatty liver disease in relation to type 2 diabetes mellitus and cardiovascular disease. Diabetes Metab Res Rev 2006;22:437-43.

11. Kim CH, Park JY, Lee KU, Kim JH, Kim HK. Association of serum gamma-glutamyltransferase and alanine aminotransferase activities with risk of type 2 diabetes mellitus independent of fatty liver. Diabetes Metab Res rev 2009;25:64-9.

12. Kim HC, Choi SH, Shin HW, Cheong JY, Lee KW, Lee HC, et al. Severity of ultrasonographic liver steatosis and metabolic syndrome in Korean men and women. World J Gastroenterol 2005;11:531421.

13. Lusong MAD, Labio E, Daez L, Gloria V. Nonalcoholic fatty liver disease in the Philippines: 
Comparable with other nations? World J Gastroenterol 2008;14:913-7.

14. Amarapurkar D, Kamani P, Patel N, Gupte P, Kumar P, Agal S, et al. Prevalence of nonalcoholic fatty liver disease: population based study. Annals of Hepatology 2007;6:161-3.

15. Schwimmer J, McGreal N, Deutsch R, Finegold MJ, Lavine JE. Influence of gender, race and ethnicity on suspected fatty liver in obese adolescents. Pediatrics 2005;115:e561-5.

16. Khurram M, Shakoor A, Arshad MM, Khaar HB, Hasan Z. Characteristic features of 50 NAFLD patients. 2004: DHQ Teaching Hospital Rawalpindi.

17. Alwis ND, Day C. Non alcoholic fatty liver disease: the mist gradually clears. J Hepatol 2008;48 Suppl 1:S104-12.

18. Shibata M, Kihara Y, Taguchi M, Tashiro M, Otsuki M. Nonalcoholic fatty liver disease is a risk factor for type 2 diabetes in middle-aged Japanese men. Diabetes Care 2007;30:2940-4.

19. Bahcecioglu IH, Koruk M, Yilmaz O, Bolukbas C, Bolukbas F, Tuncer I, et al. Demographic and clinicopathological characteristics of nonalcoholic fatty liver disease in the East Southeastern Anatolia Regions in Turkey. Med Princ Pract 2006;15:62-8.

20. Adams LA, Angulo P, Lindor KD. Nonalcoholic fatty liver disease. JAMC 2005;172:899-905.

21. Oneta CM, Dufour JF. Nonalcoholic fatty liver disease: treatment options based on pathogenic considerations. Swiss Med Wkly 2002;132:493505.

22. Olufadi R, Byrne C. Clinical and laboratory diagnosis of the metabolic syndrome. J Clin Pathol 2008;61:697-706.

23. Reynolds K, He J. Epidemiology of the metabolic syndrome. Am J Med Sci 2005;330:273-9.

24. Feldstein A, Werneburg N, Canbay A, Guicciardi M, Bronk S, Rydzewski R. Free fatty acids promote hepatic lipotoxicity by stimulating TNF- $\alpha$ expression via a lysosomal pathway. Hepatology 2004;40:185-94.

25. Chang Y, Ryu S, Sung E, Jang Y. Higher concentrations of alanine aminotransferase within the reference interval predict nonalcoholic fatty liver disease. Clin Chem 2007;53:686-92.

26. Giboney PT. Mildly elevated liver transaminase levels in the asymptomatic patient. Am Fam Physician 2005;71:1105-10.

27. Harrison SA, Tetri BN. Clinical manifestations and diagnosis of NAFLD. In: Farrell GC, George J, Hall PdlM, McCullough AJ, editors. Fatty liver disease: NASH and related disorders. $1^{\text {st }}$ ed. Massachusetts: Blackwell Publishing;2005. p.159-67.

28. Fraser A, Longnecker M, Lawlor D. Prevalence of elevated alanine aminotransferase among US adolescents and associated factors: NHANES 19992004. Gastroenterology 2007;133:1814-20.

29. Stranges S, Dorn J, Muti P, Freudenheim J, Farinaro E, Russell M, et al. Body fat distribution, relative weight and liver enzyme level: a population-based study. Hepatology 2004;39:754-63.

30. Nurman A. Non alcoholic fatty liver disease. Univ Med 2007;26:205-15.

31. Grundy SM. Gamma glutamyl transferase, another biomarker for metabolic syndrome and cardiovascular risk. Arterioscler Thromb Vasc Biol 2007;27:4-7.

32. Tiikkainen M, Bergholm R, Vehkavaara S, Aila Rissanen A, Häkkinen AM, Tamminen M, et al. Effects of identical weight loss on body composition and features of insulin resistance in obese women with high and low liver fat content. Diabetes 2003;52:701-7.

33. Thamer C, Tschritter O, Haap M, Shirkavand F, Machann J, Fritsche A, et al. Elevated serum GGT concentrations predict reduced insulin sensitivity and increased intrahepatic lipids. Horm Metab Res 2005;37:246-51.

34. Siegelman ES, Rosen MA. Imaging of hepatic steatosis. Semin Liver Dis 2001;21:71-80.

35. Mcavoy NC, Ferguson JW, Campbell ANW, Hayes PC. Non-alcoholic fatty liver disease: natural history, pathogenesis and treatment. Br J Diabetes Vasc Dis 2006;6:251-60. 